\title{
Automated Blood Glucose Control in Type 1 Diabetes: A Review of Progress and Challenges
}

Abstract: Since the 2000s, research teams around the world have been working to develop closed-loop (CL) systems able to automatically control blood glucose (BG) levels in type 1 diabetic patients. This emerging technology is known as the artificial pancreas (AP) and the first commercial version just arrived in the market. The main objective of this paper is to present an extensive review of the clinical trials from 2011 onward, which tested various implementations of the AP for different durations under varying conditions. We present a comprehensive table that contains key information from the selected publications and discuss major challenges experienced by the AP along with current mitigation strategies employed. Additionally, we present the development timelines for different AP systems highlighting the main evolutions over the clinical trials for each system.

Keywords: Artificial Pancreas; Closed-loop Glucose Control; Automated Insulin Delivery; Type 1 Diabetes Mellitus.

\section{Control Automatizado de la Glucosa en la Diabetes Tipo 1: Revisión del Progreso y los Desafíos}

Resumen: Desde los años 2000, equipos de investigación de todo el mundo han estado trabajando para desarrollar sistemas de lazo cerrado (LC) capaces de controlar automáticamente los niveles de glucosa en sangre en pacientes con diabetes tipo 1. Esta tecnología emergente se conoce como el páncreas artificial (PA) y la primera versión comercial acaba de llegar al mercado. El objetivo principal de este trabajo es presentar una extensa revisión de los ensayos clínicos realizados desde 2011, en los cuales se han puesto a prueba diversas implementaciones del AP, para diferentes duraciones y bajo diversas condiciones. Presentamos una tabla completa que contiene información clave de las publicaciones seleccionadas y se debaten los principales retos en el desarrollo del páncreas artificial, así como las estrategias de mitigación empleadas. Se 
incluye también una cronología sobre el desarrollo de los principales sistemas PA destacando las principales evoluciones en los ensayos clínicos.

Palabras clave: Páncreas artificial; Control de glucosa en lazo cerrado; Infusión automática de insulina; Diabetes mellitus tipo 1. 


\section{Introduction}

Diabetes mellitus (DM) is a widespread disease affecting approximately 415 million people worldwide and is estimated to have resulted in 5 million deaths in $2015 .{ }^{1}$ Type 1 diabetes mellitus (T1DM) affects around $5-10 \%$ of the population with diabetes. T1DM patients must be wary of both hypo- and hyperglycemia. Poor glycemic control can result in several acute and chronic complications, which can lower life expectancy or even result in death. Currently, T1DM patients control their blood glucose (BG) levels by performing treatment either using multiple daily injections (MDI) or by continuous subcutaneous insulin infusion (CSII) using an insulin pump. Patients may also use a continuous glucose monitor (CGM) in conjunction with these therapies. However, the majority of patients do not achieve tight BG control with these methods and a new approach is required to alleviate the burden of T1DM.

The artificial pancreas (AP) is a system composed of three components: a CGM, an insulin pump, and a control algorithm. Historically, the first closed-loop (CL) system designed to treat humans with T1DM dates back to the 1960's. ${ }^{2}$ The Biostator was the first commercial device to achieve tight BG control. However, it was too bulky, invasive, and complex to be used outside of a clinical environment. Now, current technology used in T1DM care are minimally invasive, lightweight, and portable. These advancements along with multiple agencies advocating for the device ${ }^{3}$ have accelerated the rate of development.

An illustration of a CL insulin delivery system is depicted in Figure 1. The main challenges identified along the development of the AP are: ${ }^{4}$ coping with large disturbances that affect the system, such as meals and exercise; creating a robust system able to adapt to the great variability of patients' physiological behavior; the inaccuracy and unreliability of continuous glucose monitors; safety of insulin pumps and detection of faults; and adequate correction for the slow responsiveness of controllers due to delays in the control loop, due especially to the response of the current insulin; and signal dropout in fully integrated systems. 


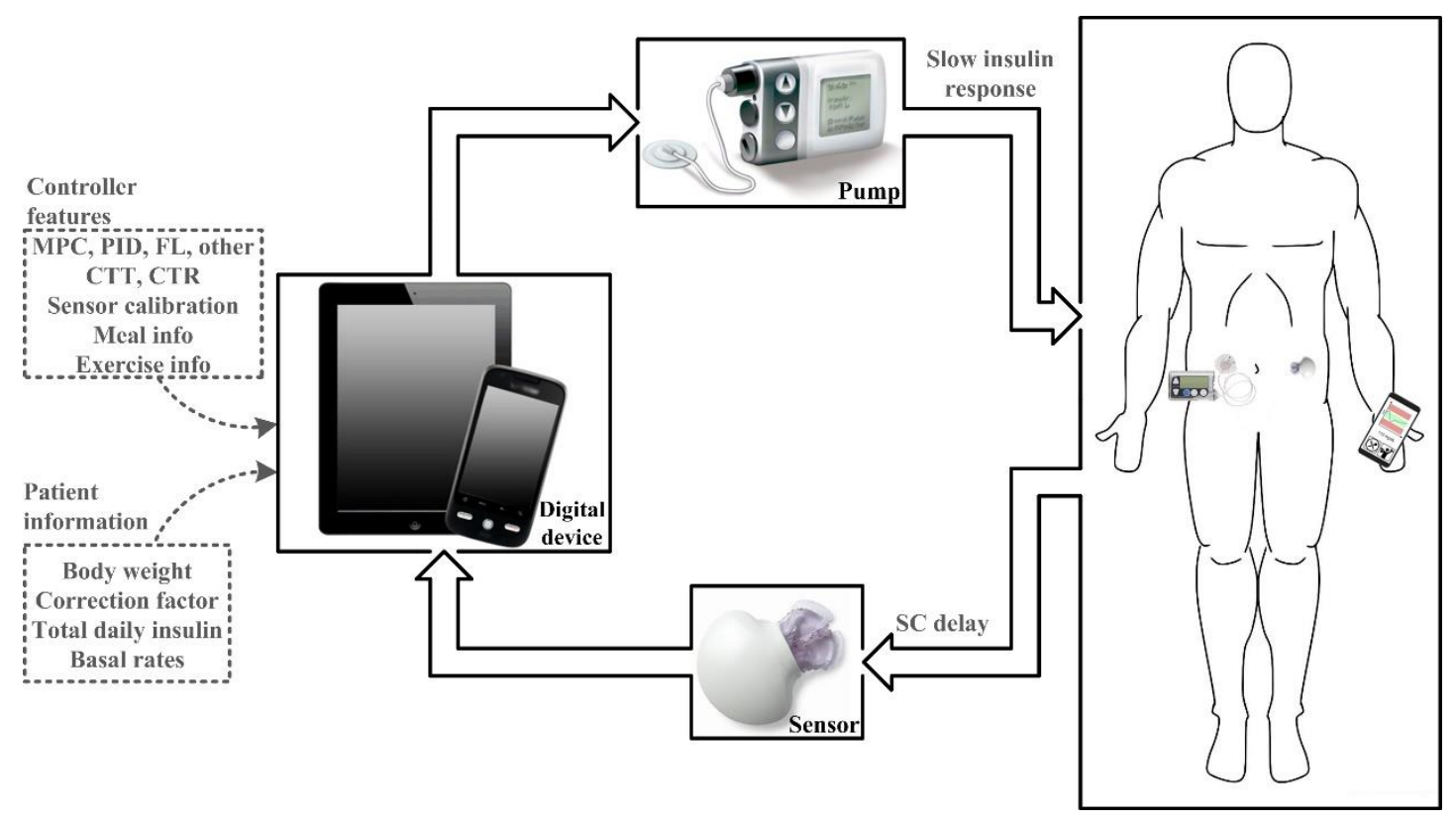

Figure 1-General closed-loop system for glucose control in type 1 diabetes mellitus. MPC: model predictive control; PID: proportional-integral-derivative; FL: fuzzy logic; CTT: control to target; CTR: control to range; SC: subcutaneous.

The rate of development of the AP is increasing rapidly and there is a fast turnover of newly published research. In 2012 and 2013, only 3 publications reported clinical studies conducted in outpatient settings. However, from 2014 onwards, the amount of outpatient trials started to increase, achieving 10 publications only in 2014 . Very recently, FDA approved the Medtronic MiniMed 670G (Northridge, CA, USA), the world's first CL system to hit the market. This system is a hybrid CL device that still requires user's actions, such as carbohydrate-counting, announcing meals and exercise. Therefore, reviews of this nature are required to inform the public of recently published papers and expose the remaining topics that still need attention to finally achieve a fully automated AP.

The main objective of this study is to present an extensive table that captures the critical information of all the clinical trials involving $C L$ systems aimed at controlling BG levels that have been performed over the last five years. This table will allow researchers quick access to information about current technologies being tested. Additionally, we present a discussion of the various challenges that remain to be overcome with regards to the AP and the evolution several systems the last years through individual timelines. 


\section{Methods}

In early October 2016, an extensive search in the US National Library of Medicine database (Pubmed.gov) for publications completed since 2011 was performed. To ensure that only clinical trials about automated BG control were analyzed, any publication in which the main objective was not the analysis of a CL system for glycemic control in humans was excluded. Publications that solely tested a low glucose suspension or insulin delivery interruption system were also excluded.

A total of 91 clinical trials were included in this review. For each publication, we extracted information and grouped them into three subgroups: system features, scenario features, and CL outcomes. In the field intended for comments (see Table 1, Supplementary Material 1), in some cases, there is also a classification for the different arms within the same publication. Outcomes ending in (A) relate to the results of arm 1 and those that end with $(B)$ are related to arm 2, and so on.

In the system features group, four features were extracted:

- Controller characteristics: classifies each controller tested into 3 classes of control algorithms: Proportional-Integral-Derivative (PID), Model Predictive Controller (MPC) and Fuzzy Logic (FL). Other strategies were classified as "Other". Control algorithms are further separated into either Control-To-Range (CTR) or Control-to-Target (CTT) strategy.

- Meal bolus: reports publications that used insulin boluses to cover meals (i.e., meal announcement).

- Dual-hormone: reports systems that include insulin and glucagon delivery.

- Fully integrated: reports systems with automated data transfer from the CGM to the controller and from the controller to the insulin pump, without manual action.

Regarding the scenario description, five characteristics are presented for each publication.

- Cohort: age group and number of subjects tested in $\mathrm{CL}$ therapy. Patients who did not complete the full study, were not counted. 
- Challenges: scenarios under which the controller's performance was challenged, such as, meal, exercise, stress, alcohol, pregnancy, and free-living conditions.

- Closed-loop duration: the total duration of CL operation reported in each publication.

- Overnight: reports $\mathrm{CL}$ systems only tested during the overnight period. It may include dinner and/or breakfast meals, but not a lunch meal.

- Outpatient: studies performed partially or completely outside of a clinical research center. It also includes whether the outpatient trial was conducted with remote monitoring $(R M)$, under supervision (SU), or with neither remote monitoring nor supervision (NRS).

And finally, mean glucose levels during the CL therapy, percent of time spent in euglycemic range $(70-180 \mathrm{mg} / \mathrm{dL})$ and in hypoglycemic range $(<70 \mathrm{mg} / \mathrm{dL})$ are also shown when available, as $\mathrm{CL}$ outcomes. These outcomes are presented as mean values and within the specific glucose ranges in $\mathrm{mg} / \mathrm{dL}$, unless stated otherwise.

\section{Results}

An agglomerate of features collected from the 91 publications are presented in Table 1 (see Supplementary Material 1), sorted by year of publication. We have conducted a general analysis of the clinical trials, to identify advances, possible gaps, and problems that are yet to be addressed in CL systems for T1DM treatment. It is important to note that it is not our objective to rank the trials performed and we understand that it is not possible to compare the performance between these controllers, due to the multitude of differences between each protocol, study methods, and objectives.

The evolution of different systems in development, ${ }^{5}$ is presented in individual timelines (see Supplementary Material 2). Through these timelines it is possible to notice that the trials evolved from very controlled conditions to trials that aimed to evaluate these systems as close as possible to real life. 


\section{Discussion}

Several technological advancements in AP technology have taken place since the publication of Steil et al.., ${ }^{6}$ which showed the feasibility of automated insulin delivery to treat T1DM. Formerly, systems were tested on patients in a controlled clinical environment, only during the night and even tested in non-human subjects. Now, some publications have been performed under free-living conditions without any kind of remote monitoring or supervision by clinical staff. Indeed, for a final realization of commercial AP devices, CL systems must be challenged under real-life conditions.

There are three main types of control strategies that have been tested: proportionalintegral-derivative (PID), model predictive control (MPC) and fuzzy logic (FL). Albeit other algorithms can been seen, such as the USS Virginia, ${ }^{7}$ based on future predictable risks of hypoor hyperglycemia, MPC was the most used strategy in the trials (56\%), followed by the PID (28\%). FL controller was used only in $7 \%$ of the trials and other strategies in $9 \%$. A review of the stateof-the-art in control algorithms for BG control can be found elsewhere. ${ }^{8}$

Although, many different controllers for AP systems have been tested clinically many difficulties still arise and there is great room for improvement of control algorithms. The following subsections will differentiate between challenges that have been extensively addressed versus challenges that have been minimally investigated.

\subsection{Extensively Addressed Challenges}

Initially, the suspension of insulin administration to prevent nocturnal hypoglycemia was utilized by CGM--CSII software algorithms. Trials have since then evolved to be fully integrated systems that include manual and automated strategies to prevent postprandial hyperglycemia due to meals, exercise-induced hypoglycemia, additional hormones (such as glucagon and pramlintide) and hardware malfunctions. In the next sections advancements in control algorithms to deter these effects will be discussed. 


\subsubsection{Overnight}

Prevention of nocturnal hypoglycemia is critical in those with T1DM. Presently, AP systems have been shown to be robust and effective at preventing nocturnal hypoglycemia. One study by Phillip et al. ${ }^{9}$ found a $32 \%$ decrease in the amount of nocturnal hypoglycemic events. Russell et al. ${ }^{10}$ also reported a $94 \%$ decrease in glucose levels below $60 \mathrm{mg} / \mathrm{dL}$ during the overnight period using a dual-hormone controller, and Thabit et al. ${ }^{11}$ reported that children/adolescents and adults during the nighttime period spent $24.7 \%$ and $19.4 \%$ more time in the target range $(70-145 \mathrm{mg} / \mathrm{dL})$, respectively. Outcomes of these studies were all compared to sensor-augmented pump (SAP) therapy.

However, it was also noted by these studies ${ }^{10,11}$ that the benefits of CL control was seen largely during overnight periods as compared to daytime periods where large glucose fluctuations due to meals and exercise occur.

\subsubsection{Meals}

Meals can be considered the major cause of glucose variability in T1DM. ${ }^{12}$ In all T1DM studies, meals induce changes in glucose levels during the intervention period. Although in some studies the CL system operates only overnight, evening meals can induce glucose variability for this period.

Currently, either one of two strategies are used to handle meals in CL systems: 1) hybrid CL systems, where the user announces the meal time and $\mathrm{CHO}$ content (i.e. feedforward action) or 2) fully CL systems, where the system performs control of BG without meal boluses, sometimes, using a meal detection algorithm.

Hybrid CL systems use insulin boluses to control glucose variations due to meals, in Table 1 (Supplementary Material 1) this is indicated by a tick in the "Meal Bolus" column. A premeal insulin bolus is able to reduce postprandial glycemic excursions, with optimal results obtained with the bolus delivery 15 minutes before meals. ${ }^{13}$ The bolus is calculated using the user-informed 
$\mathrm{CHO}$ content of meals and the insulin-to-carbohydrate ratio of the patient. However, this strategy is a burden to users and does not guarantee optimal control, since subjects generally underestimate $\mathrm{CHO}$ content with $20.9 \%$ as medium error. ${ }^{14}$ In fully $\mathrm{CL}$ systems, the controller is responsible for detecting glucose variations and making corrections automatically. Additionally, it has been found that meals high in fat and protein require more insulin to control late postprandial hyperglycemia. ${ }^{15}$ Several algorithms have been recently developed to detect meals, ${ }^{16,17}$ which allow a controller to employ a feedback system to reduce postprandial hyperglycemia.

The two main drawbacks to control during the postprandial period are attributed to the delayed CGM readings due to the interstitial-plasma time lag and the delay in insulin action. A significant challenge to the AP, inherent to the CGM is the time required for glucose to diffuse from the blood into the interstitial space, where the CGM probe is inserted. Current CGM technology inherently under- or overestimate BG levels because the BG concentration found in the interstitial fluid is not a perfect reflection of the BG concentration in the plasma. ${ }^{18}$ Therefore, calibration with the aid of a glucose meter performed at regular intervals is required to correct these inaccuracies. ${ }^{19}$

The delay experienced between the infusion and the onset of current insulin is a hurdle for automatic systems to properly control blood glucose levels during the postprandial period. Currently "fast-acting" is not fast enough to achieve optimal postprandial control in fully CL systems, that do not require meal announcement to cover meals. Ultra-fast or ultra-rapid insulin produces a faster onset of insulin action, which better mimics the first-phase insulin response in non-diabetic people. ${ }^{20}$

An approach widely studied by researchers recently is the addition of a glucagon pump to the system that uses glucagon to counterbalance insulin, which mimics the behavior of pancreatic a cells in healthy subjects. In contrast to insulin which lowers glucose, glucagon elevates BG levels through hepatic processes. With this approach, $C L$ systems can be more aggressive with BG control, especially after meals. However, some also argue that the use of glucagon 
aggressively is not biologically feasible. Additional limitations of glucagon are discussed further in Section 4.1.4.

The use of pramlintide and exenatide have also been investigated to improve postprandial glycemia together with CL therapy. The use of these adjunctive therapies suggest a reduction in postprandial peaks and thereafter in insulin overdosing. In Weinzimer et al. ${ }^{21}$ patients received pramlintide subcutaneously at meal time and no meal boluses were delivered to cover meals. Authors noticed that pramlintide delayed the time from meal peak approximately by 1 hour and also decreased glucose excursions by an average of $25 \mathrm{mg} / \mathrm{dL}$, when compared with insulin alone. Similar results were obtained by Sherr et al., ${ }^{22}$ where they observed an average reduction of $37 \mathrm{mg} / \mathrm{dL}$ in glucose excursions after meals, and also a significant delay in glucose meal peak due to pramlintide. In Renukuntla et al., ${ }^{23}$ patients received different treatments to study the effects of short-term use of pramlintide and exenatide in CL systems. Their results showed a better performance of exenatide in postprandial glucose excursions. When compared with pramlintide, the adjunctive treatment with exenatide reduced the time spent in hyperglycemia (>180 mg/dL), $16 \%$ vs. $28 \%$. Although the results have shown better results than insulin only therapy, in both studies the hormones were injected manually and further research is required before these hormones can be used in CL systems.

\subsubsection{Exercise}

It is well established that exercise is beneficial in healthy subjects. Meanwhile, for individuals with T1DM, exercise creates a paradox by increasing overall health and fitness but makes the management of blood glucose control more difficult.

Notifying the controller with information regarding physical activity (e.g. activity type, expected duration, intensity) based on a similar strategy used with meals could improve the controller's ability to keep BG values within a specified target. This announcement allows changes in controller's aggressiveness and glucose target during this period of exercise. 
Different approaches to prevent exercise-induced hypoglycemia have been tested recently in clinical trials. In Ly et al., ${ }^{24}$ the target glucose was set at $120 \mathrm{mg} / \mathrm{dL}$, but when exercise was announced, the target glucose increased to $160 \mathrm{mg} / \mathrm{dL}$. This study was able to decrease the percentage of time spent in hypoglycemia from $7.6 \%$ in the SAP to $2.0 \%(p=0.013)$. In Blauw et al., ${ }^{25}$ heart rate was used to indicate physical activity, which reduced insulin administration during that period. This study showed a non-significant decrease in the percentage of time spent below $70 \mathrm{mg} / \mathrm{dL}$ from $2.4 \%$ in the conventional therapy to $1.3 \%(p=0.139)$ in $\mathrm{CL}$ therapy. In Jacobs et al., ${ }^{26}$ a dual-hormone controller reduced insulin and increased glucagon delivery for 90 minutes after the start of exercise. This study also obtained non-significant results with a decrease in the percentage of time spent below $70 \mathrm{mg} / \mathrm{dL}$ from $0.8 \%$ in the SAP to $0.3 \%(p=0.16)$ in the $C L$ period.

Physical activity detection in real time has been investigated ${ }^{27}$ and can be a useful tool that will minimize the error attributed to patient inputs (previously described in the meal section above). Various studies ${ }^{25,28-30}$ have incorporated physical signals (e.g. Heart Rate, Energy Expenditure, Galvanic Skin Response) into the controller during exercise, and although this approach may increase controller performance, ${ }^{29}$ it also increases the overall complexity and cost of the system.

Although exercise has been extensively investigated, much work still remains. The effects of exercise are not fully understood, with exercise having an extended effect on BG. Additionally, exercise has yet to be implemented into the current simulators, limiting in silico trials of this nature.

\subsubsection{Dual-hormone}

Dual-hormone systems including insulin and glucagon have been tested, however it is often reported that due to the instability of glucagon, cartridges must be frequently replaced. Currently, there is no stable glucagon solution commercially available so far, although research has been conducted to overcome this, ${ }^{31}$ nor are there dual-chamber pumps, studies presently use two pumps which can be bulky and lead to patient dissatisfaction..$^{32}$ It is expected that when dual-hormone systems reach the market, dual-chamber pumps will be already developed. 
Different approaches with regard to glucagon delivery have been assessed: limiting the total glucagon delivery to a specified amount for a given period of time ${ }^{33,34}$ or delivering glucagon without this restriction, ${ }^{35}$ suspending insulin infusion while glucagon is being delivered ${ }^{36}$ or delivering both hormones at the same time. ${ }^{37}$ Although it is a likely solution for hypoglycemia prevention, it is not yet clear how effective this approach will be. The first head-to-head comparison between single- and dual-hormone systems was published in $2015 .{ }^{38}$ During 24 hours in an inpatient setting, participants were engaged to simulate outpatient conditions with meals, social activities and 60 minutes of exercise on a treadmill. Both CL systems outperformed conventional insulin therapy in this study. Patients spent less time in hypoglycemia $(<72 \mathrm{mg} / \mathrm{dL})$ with dual-hormone than with the single-hormone therapy, $1.5 \%$ vs. $3.1 \%(p=0.018)$, respectively. However, overnight results showed that single-hormone therapy might be enough to eliminate hypoglycemia during overnight.

Another head-to-head comparison between a dual- and single-hormone therapy during exercise sessions was assessed by Taleb et al. ${ }^{39}$ Two different exercise sessions were performed by 17 adults on a ergocycle: continuous exercise for 60 minutes and interval session for 40 minutes with alternations in the intensity. Exercise was announced 20 minutes earlier for an MPC controller responsible for insulin calculation and glucagon was delivered based on logic rules. During continuous exercise nine participants had hypoglycemia ( $<70 \mathrm{mg} / \mathrm{dL})$ in single-hormone therapy vs. three participants with the dual-hormone therapy $(p=0.07)$. Similarly, less patients experienced hypoglycemia in dual-hormone arm during interval exercise: one patient vs. seven in single-hormone arm $(p=0.04)$.

In the studies performed thus far, glucagon has been able to reduce time spent in hypoglycemia. Larger and longer studies are still required to evaluate the results of dual-hormone systems in $24 \mathrm{~h}$ operation, ${ }^{40}$ as well as the side-effects and risks of long-term utilization must be thoroughly investigated. The development of new insulin formulation, such as ultra-rapid insulin, might eliminate or reduce the requirement of glucagon in future $\mathrm{CL}$ systems. ${ }^{41}$ Until then, dual- 
hormone systems can potentially increase the safety of CL systems and counteract the slow response of current insulin formulations.

\subsubsection{Hardware reliability}

As with all electromechanical systems, the AP is susceptible to malfunctions. The AP is intended to be a fully integrated system, therefore, it is essential that those designing the system be aware of the kinds of failures that can occur, to ensure the safety of the users at all times.

Bequette $^{42}$ presents an evaluation of failures that can occur in an AP and indicates that CGM anomalies (fouling of sensors, loss of signal, signal degradation) and the insulin infusion set failures are the two most frequent hardware-based failures. Heinemann and Krinelke ${ }^{43}$ have said that the insulin infusion set is the main culprit of CSII malfunctions and often cause users to abandon pump therapy. ${ }^{44}$ Blauw et al. ${ }^{45}$ features an extended review of design requirements to improve safety in the AP, not only considering hardware failures, but also other situations such as cybersecurity and issues related with the control algorithm.

Although the use of CGMs is associated with a reduction in $\mathrm{HbA1c},{ }^{46}$ the reliability of CGMs is still a problem for CL strategies. Data flow between the transmitter and the receiver of a CGM is wireless and data can be intermittently missed. Schmidt et al. ${ }^{47}$ reported that $5.5 \%$ of CGM data during their clinical trial were missed (196 of 3564 values). Therefore, because of this known lack of data the control algorithm must be designed to utilize past CGM measures or predict future CGM values for insulin administration decisions.

We observed a lack of information on fault detection systems in the studies analyzed. Few authors have reported concern about faults in the system design. In fully integrated systems, a high incidence of problems related to pump connectivity were reported as being responsible for more than $50 \%$ of $\mathrm{CL}$ interruption in some cases. ${ }^{11}$

To follow the natural evolution from inpatient to outpatient settings, a large increase in the number of fully integrated systems was observed. In 2011 , only $29 \%$ of the publications report 
the use of fully integrated systems, in contrast to 2015 where $85 \%$ of the systems tested were fully integrated. Several different types of devices have been used to embed the firmware required, such as laptops, tablets and different brands of mobile phones. Dedicated platforms have been designed to combine all the devices and includes a user interface, such as the Diabetes Assistant (DiAs) and the FlorenceD2A, developed by the University of Virginia and by the University of Cambridge, respectively. In the system used by Ly et al. ${ }^{48}$ the algorithm was incorporated within the insulin pump, which eliminates the persistent problem of pump connectivity faced by other groups. Communication interruption between devices increases the probability of system faults and failures and can affect the behavior of the entire system which ultimately, increases the user-related risk. ${ }^{45}$

\subsection{Minimally Investigated Challenges}

In contrast to meals and exercise, several challenges that can have severe impacts on the AP have not been intimately investigated. These challenges include alcohol, pregnancy, user error, inclusion of patients with high levels of $\mathrm{HbA1c}$, additional concomitant diseases and of varying ages.

\subsubsection{Alcohol}

In general, when T1DM patients consume alcohol without adjusting insulin therapy ${ }^{49}$ their risk of hypoglycemia is increased. It has been reported that the delayed effect associated with reduced nocturnal growth hormone secretion after alcohol consumption ${ }^{50}$ can lead to next-day hypoglycemic events. ${ }^{51}$

In the results presented in Table 1 (see Supplementary Material 1), five studies allowed alcohol consumption 7,10,52-54 (disregarding any publication defined as "free-living conditions"). In Hovorka et al., ${ }^{52}$ subjects had dinner accompanied by wine, afterwards the CL system maintained BG levels overnight. Based on the study's outcomes, the authors concluded that alcohol did not affect the CL performance in their study. In Russell et al. ${ }^{10}$ adults were allowed to consume up to 3 alcoholic drinks per day. In a free-living study by Van Bon et al. ${ }^{55}$ some patients reported that 
they consumed alcoholic drinks in social gatherings, and that it did not influence CL performance. Due to protocol peculiarities, none of the aforementioned studies presented detailed information on alcohol consumption nor analyzed the isolated effect of alcohol consumption on AP behavior.

It has been found that heavy alcohol consumption contributes to disease complications and may temporarily reduce patient competence with respect to performing critical tasks related to self-treatment, such as, bolus dosing and CGM calibration. ${ }^{56}$ This effect further challenges the controller and reduces the patients ability to perform important tasks related to AP operation, especially in hybrid $C L$ systems.

\subsubsection{Pregnancy}

A complete understanding about the effects of pregnancy in women with T1DM is not known. ${ }^{12}$ During early pregnancy, women spend almost $15 \%$ of time below $70 \mathrm{mg} / \mathrm{dL}$ and $5.7 \%$ below $50 \mathrm{mg} / \mathrm{dL}$, in severe hypoglycemia. ${ }^{57}$ Despite the short duration and inpatient procedures, two studies published by Murphy and colleagues ${ }^{58,59}$ showed that glucose control in pregnant women is improved with CL therapy. Since those studies published in 2011, just one more trial was reported with pregnant patients. ${ }^{60} \mathrm{CL}$ therapy outperformed SAP therapy in mean glucose 119 vs. $133 \mathrm{mg} / \mathrm{dL}$ ( $p=0.009$ ), as well as reduced time spent in hypoglycemia during overnight control for 4 weeks.

Pregnant women with T1DM require tight glucose control to prevent unwanted outcomes. This subset of patients should not be neglected and a specialized controller solely for this population should be considered. An adaptive control strategy may be requested for this special group of type 1 patients due the continuous changes of insulin requirement during pregnancy. Dual-hormone systems have not been tested in this group of diabetic patients, however, sideeffects of glucagon, such as vomiting and nausea, may be more evident in this group due to gastrointestinal sensitivity experienced during pregnancy.

\subsubsection{Controllers across ages}


We have observed that several papers have not specified the amount of subjects that were adolescents, children, or adults but rather reported the total number of subjects only stating which subgroups were involved. A longitudinal study of healthy children aged 5-14 years found that the rise in insulin resistance occurs 3-4 years prior to the onset of puberty and appears to be closely related to age. ${ }^{61}$ Therefore, the control during childhood and adolescence is more challenging and this information is vital when comparing controller performance and robustness between age groups. El-Khatib et al. ${ }^{37}$ reported that the second generation of their bionic pancreas could not provide good performance in adolescents when set for adults and vice versa, due to different insulin requirements between groups.

Additionally, few studies tested their system for adults (AD), adolescents $(A L)$ and children $(\mathrm{CH})$ under the same protocol. In Nimri et al. ${ }^{62}$ a total of 12 subjects (4 AD, $4 \mathrm{AL}$ and $4 \mathrm{CH}$ ) spent the night in the clinic under CL overnight control. In this trial, there were no hypoglycemic event in CL therapy vs. three in open-loop treatment $(p=0.18)$. The authors did not comment about the influence of patient's ages in the CL performance. In Nimri et al. ${ }^{63}$ a 6-week overnight CL study was performed in $\mathrm{AD}, \mathrm{AL}$ and $\mathrm{CH}$. Results showed that overnight mean $\mathrm{BG}$ was significantly reduced during CL when compared with SAP, $148 \mathrm{mg} / \mathrm{dL}$ vs. $161 \mathrm{mg} / \mathrm{dL}(p=0.008)$ and the time spent in hypoglycemic range was reduced by $1.86 \%(\mathrm{p}=0.02)$ in $\mathrm{CL}$ nights. Again, the authors did not comment on the influence of patient age on the outcomes.

The results of the above-mentioned studies evidence that these systems are suitable for $\mathrm{AD}, \mathrm{AL}$ and $\mathrm{CH}$ during overnight periods, although an analysis of data between age groups would be useful. One point of note is that in free-living conditions, daily activities between these age groups are distinct and the reported averaged outcomes may not be acceptable over longer CL durations.

\subsubsection{Inclusion/Exclusion Criteria}

To improve the efficiency for trials with small groups the FDA recommends that clinical trials should include subjects with high $\mathrm{HbA} 1 \mathrm{c}$ levels, however it was observed that only 18 studies 
reported $\mathrm{HbA} 1 \mathrm{c}$ values (mean or median) higher than $8 \%$. Moreover, clinical trials are designed in such a way that patients with high $\mathrm{HbA1c}$ levels are not able to participate due to inclusion/exclusion criteria. In 2012, the T1D Exchange Clinic Network ${ }^{64}$ published data of over 25000 T1DM patients, $52 \%$ of those participants had $\mathrm{HbA} 1 \mathrm{c}$ levels equal to or higher than $8 \%$. In the studies analyzed, the highest mean $\mathrm{HbA1c}$ reported was 9.2 (1.2) \% in a study involving 12 adolescents. ${ }^{65}$

Several conditions associated with T1DM are usually included in the exclusion criteria for AP trials. These include hypoglycemia unawareness, thyroid disease, hypertension, and microvascular complications such as nephropathy, retinopathy, and neuropathy. In fact, almost all trials included in this review excluded those with one or more of these conditions.

Excluding patients with high HbA1c levels and additional diseases and complications common to those with T1DM excludes a large population of those with T1DM from trials. These patients are arguably the population that will benefit the most from AP therapy.

$\mathrm{HbA1c}$ represents the average BG levels over a 3-month period but does not provide information about glucose variability. Currently, $\mathrm{HbA} 1 \mathrm{c}$ is the most accepted metric used to determine the likelihood of long-term complications. ${ }^{66}$ Long-term trials are able to assess the improvement of $\mathrm{HbA} 1 \mathrm{c}$ in $\mathrm{CL}$ studies. In our results, we observed that three publications reported a reduction of $\mathrm{HbA1c} .^{11,67,68}$ Reduction in $\mathrm{HbA} 1 \mathrm{c}$ coupled with a reduced percentage of time in the hypoglycemic range is a good indicator of the efficacy of a CL system, allowing it to reduce the risk of long-term complications without increasing the amount of hypoglycemic events.

\subsubsection{Comparison Between Controllers Performance}

A fair comparison between controllers is possible only when they are tested under the same conditions, in other words, the controllers must be tested in the same patients and follow the same protocol. This is made even more challenging as many research groups around the world are developing novel methods and strategies to cope with the various challenges invoked by T1DM. 
Pinsker et al. ${ }^{69}$ performed an outpatient study designed to compare the performance of the two most widely tested control algorithms, the MPC and PID, under realistic situations. The controllers were challenged by one unannounced and two announced meals, including an overnight period. The overall outcomes showed that MPC spent more time in range (70-180 $\mathrm{mg} / \mathrm{dL})$ than PID, $74.4 \%$ vs. $63.7 \%(p=0.021)$, respectively. However, any comparison is specific for the particular tuning of the controllers, and it is not possible to generalize that all MPC controllers will be better than PID controllers. In the aforementioned study, authors concluded that both controllers are suitable for glycemic control, providing safety for the users.

We have found that many studies used a non-standardized time in range and time in hypoglycemia to report their outcomes, which makes comparison of controller performance difficult. This observation was also noted by Doyle et al. ${ }^{70}$ Basic outcome measures should be reported by AP clinical trials, this will make the comparison between future outpatient studies in free-living conditions feasible. ${ }^{66}$

\subsubsection{User Error}

Current CGMs and CSII pumps require frequent intervention by the patient or a caregiver. CGM sensors must be calibrated often (up to 2 times daily) and replaced every 7-14 days; and the insulin cartridge and infusion set must be replaced frequently. These replacements, if not done correctly can lead to infection and further complications. Sensor miscalibrations can lead to erroneous CGM readings, causing incorrect insulin dosing. We found that in many of the outpatient studies conducted, the research team provided beforehand technical training on device operation to the participants. This training is beneficial and reduces the risk associated with patient error, but does not eliminate it. Further studies investigating the impact of hazardous situations on the AP are needed to test the safety limits of the device.

Different levels of automation can be observed in the systems tested. The so-called "hybrid" CL systems that utilize feedforward action require the patient to report known external events, such as meal and exercise information, which improve the system's performance. This 
kind of AP system is susceptible to human error, which can ultimately compromise its performance. Alternatively, fully CL systems that utilize feedback action require no intervention from the patient and are able to perform necessary modifications to overcome external disturbances. However, due to the slow response of insulin after delivery and the interstitialplasma time lag experienced by CGMs, feedback systems perform poorly in the postprandial period.

\section{Conclusions}

The first generation of commercial APs is already in the market. Landmark clinical study results have reinforced the AP's ability to improve glycemic control without increasing the risk of hypoglycemia when compared head-to-head with the current state-of-the- art, i.e. SAP therapy. T1DM patients using these first commercial CL devices will still have to be vigilant, but the burden of their disease will be significantly lessened.

Various scenarios have been tested throughout the years to challenge different types of controllers, and in most cases, better outcomes have been obtained in CL delivery when compared to open-loop and SAP therapy. However, difficult situations such as very large meals, high intensity exercise, high stress environments, and heavy alcohol consumption have yet to be explored and their effects on CL systems are unknown.

Moreover, it is necessary to overcome challenges that involve intensive and prolonged use of a complex system, which contains multiple components with different requirements and lifespans, managed by humans with minimal training, and a tendency to non-adherence. The constant challenges of efficacy and safety enter a new dimension when considering long periods of time, in which many actions must be carried by the patient without supervision.

It is an exciting time for the development of the AP especially with the recent launch of the MiniMed 670G, a hybrid CL system. However, many challenges still remain and need to be faced by developers, especially for fully automated systems. 


\section{References}

1. International Diabetes Federation. IDF Diabetes Atlas, 7th edn. [Internet]. 2015. Available from: http://www.diabetesatlas.org

2. Cobelli C, Renard E, Kovatchev B. Artificial Pancreas: Past, Present, Future. Diabetes. $2011 ; 60(11): 2672-82$.

3. Kowalski A, Lum JW. Juvenile Diabetes Research Foundation Artificial Pancreas Consortium Update. J Diabetes Sci Technol. 2009;3(5):1224-6.

4. Hovorka R. The Future of Continuous Glucose Monitoring: Closed Loop. Curr Diabetes Rev. 2008;4(3):269-79.

5. Trevitt S, Simpson S, Wood A. Artificial Pancreas Device Systems for the Closed-Loop Control of Type 1 Diabetes: What Systems Are in Development? J Diabetes Sci Technol. 2016;10(3):714-23.

6. Steil GM, Rebrin K, Darwin C, Hariri F, Saad MF. Feasibility of Automating Insulin Delivery for the Treatment of Type 1 Diabetes. Diabetes. 2006;55(12):3344-50.

7. Brown SA, Kovatchev BP, Breton MD, Anderson SM, Keith-Hynes P, Patek SD, et al. Multinight "Bedside" Closed-Loop Control for Patients with Type 1 Diabetes. Diabetes Technol Ther. 2015;17(3):203-9.

8. Lunze K, Singh T, Walter M, Brendel MD, Leonhardt S. Blood glucose control algorithms for type 1 diabetic patients: A methodological review. Biomed Signal Process Control. 2013;8(2):107-19.

9. Phillip M, Battelino T, Atlas E, Kordonouri O, Bratina N, Miller S, et al. Nocturnal Glucose Control with an Artificial Pancreas at a Diabetes Camp. N Engl J Med. 2013;368(9):82433.

10. Russell SJ, El-Khatib FH, Sinha M, Magyar KL, McKeon K, Goergen LG, et al. Outpatient 
Glycemic Control with a Bionic Pancreas in Type 1 Diabetes. N Engl J Med. 2014;371(4):313-25.

11. Thabit H, Lubina-Solomon A, Stadler M, Leelarathna L, Walkinshaw E, Pernet A, et al. Home use of closed-loop insulin delivery for overnight glucose control in adults with type 1 diabetes: a 4-week, multicentre, randomised crossover study. Lancet Diabetes Endocrinol. 2014;2(9):701-9.

12. Kudva YC, Carter RE, Cobelli C, Basu R, Basu A. Closed-Loop Artificial Pancreas Systems: Physiological Input to Enhance Next-Generation Devices. Diabetes Care. 2014;37(5):1184-90.

13. Luijf YM, van Bon AC, Hoekstra JB, DeVries JH. Premeal Injection of Rapid-Acting Insulin Reduces Postprandial Glycemic Excursions in Type 1 Diabetes. Diabetes Care. 2010;33(10):2152-5.

14. Brazeau AS, Mircescu H, Desjardins K, Leroux C, Strychar I, Ekoé JM, et al. Carbohydrate counting accuracy and blood glucose variability in adults with type 1 diabetes. Diabetes Res Clin Pract. 2013;99(1):19-23.

15. Bell KJ, Smart CE, Steil GM, Brand-Miller JC, King B, Wolpert HA. Impact of Fat, Protein, and Glycemic Index on Postprandial Glucose Control in Type 1 Diabetes: Implications for Intensive Diabetes Management in the Continuous Glucose Monitoring Era. Diabetes Care. 2015;38(6):1008-15.

16. Turksoy K, Samadi S, Feng J, Littlejohn E, Quinn L, Cinar A. Meal Detection in Patients With Type 1 Diabetes: A New Module for the Multivariable Adaptive Artificial Pancreas Control System. IEEE J Biomed Heal Informatics. 2016 Jan;20(1):47-54.

17. Harvey RA, Dassau E, Zisser H, Seborg DE, Doyle FJ. Design of the Glucose Rate Increase Detector: A Meal Detection Module for the Health Monitoring System. J Diabetes Sci Technol. 2014;8(2):307-20. 
18. Del Favero S, Facchinetti A, Sparacino G, Cobelli C. Improving Accuracy and Precision of Glucose Sensor Profiles: Retrospective Fitting by Constrained Deconvolution. Biomed Eng IEEE Trans. 2014;61(4):1044-53.

19. Tumminia A, Sciacca L, Frittitta L, Squatrito S, Vigneri R, Le Moli R, et al. Integrated insulin pump therapy with continuous glucose monitoring for improved adherence: technology update. Patient Prefer Adherence. 2015;9:1263-70.

20. Danne T, Bolinder J. New Insulins and Insulin Therapy. Diabetes Technol Ther. 2014;16(S1):S34-43.

21. Weinzimer SA, Sherr JL, Cengiz E, Kim G, Ruiz JL, Carria L, et al. Effect of Pramlintide on Prandial Glycemic Excursions During Closed-Loop Control in Adolescents and Young Adults With Type 1 Diabetes. Diabetes Care. 2012;35(10):1994-9.

22. Sherr JL, Patel NS, Michaud Cl, Palau-Collazo MM, Van Name MA, Tamborlane W V, et al. Mitigating Meal-Related Glycemic Excursions in an Insulin-Sparing Manner During Closed-Loop Insulin Delivery: The Beneficial Effects of Adjunctive Pramlintide and Liraglutide. Diabetes Care. 2016;39(7):1127-34.

23. Renukuntla VS, Ramchandani N, Trast J, Cantwell M, Heptulla RA. Role of Glucagon-Like Peptide-1 Analogue Versus Amylin as an Adjuvant Therapy in Type 1 Diabetes in a Closed Loop Setting With ePID Algorithm. J Diabetes Sci Technol. 2014;8(5):1011-7.

24. Ly TT, Weinzimer SA, Maahs DM, Sherr JL, Roy A, Grosman B, et al. Automated hybrid closed-loop control with a proportional-integral-derivative based system in adolescents and adults with type 1 diabetes: individualizing settings for optimal performance. Pediatr Diabetes. 2017;18(5):348-55.

25. Blauw $\mathrm{H}$, van Bon AC, Koops R, DeVries $\mathrm{JH}$. Performance and safety of an integrated bihormonal artificial pancreas for fully automated glucose control at home. Diabetes, Obes Metab. 2016;18(7):671-7. 
26. Jacobs PG, El Youssef J, Reddy R, Resalat N, Branigan D, Condon J, et al. Randomized trial of a dual-hormone artificial pancreas with dosing adjustment during exercise compared with no adjustment and sensor-augmented pump therapy. Diabetes, Obes Metab. 2016;18(11):1110-9.

27. Turksoy K, Paulino TML, Zaharieva DP, Yavelberg L, Jamnik V, Riddell MC, et al. Classification of Physical Activity: Information to Artificial Pancreas Control Systems in Real Time. J Diabetes Sci Technol. 2015;9(6):1200-7.

28. Turksoy K, Bayrak ES, Quinn L, Littlejohn E, Cinar A. Multivariable Adaptive Closed-Loop Control of an Artificial Pancreas Without Meal and Activity Announcement. Diabetes Technol Ther. 2013;15(5):386-400.

29. Breton MD, Brown SA, Karvetski CH, Kollar L, Topchyan KA, Anderson SM, et al. Adding heart rate signal to a control-to-range artificial pancreas system improves the protection against hypoglycemia during exercise in type 1 diabetes. Diabetes Technol Ther. 2014;16(8):506-11.

30. Turksoy K, Quinn LT, Littlejohn E, Cinar A. An Integrated Multivariable Artificial Pancreas Control System. J Diabetes Sci Technol. 2014;8(3):498-507.

31. Pohl R, Li M, Krasner A, De Souza E. Development of Stable Liquid Glucagon Formulations for Use in Artificial Pancreas. J Diabetes Sci Technol. 2015;9(1):8-16.

32. Barnard KD, Wysocki T, Thabit H, Evans ML, Amiel S, Heller S, et al. Psychosocial aspects of closed- and open-loop insulin delivery: closing the loop in adults with Type 1 diabetes in the home setting. Diabet Med. 2015;32(5):601-8.

33. El Youssef J, Castle JR, Branigan DL, Massoud RG, Breen ME, Jacobs PG, et al. A controlled study of the effectiveness of an adaptive closed-loop algorithm to minimize corticosteroid-induced stress hyperglycemia in type 1 diabetes. J Diabetes Sci Technol. $2011 ; 5(6): 1312-26$. 
34. Van Bon AC, Jonker LD, Koebrugge R, Koops R, Hoekstra JBL, DeVries JH. Feasibility of a bihormonal closed-loop system to control postexercise and postprandial glucose excursions. J Diabetes Sci Technol. 2012;6(5):1114-22.

35. Haidar A, Rabasa-Lhoret R, Legault L, Lovblom LE, Rakheja R, Messier V, et al. Singleand Dual-Hormone Artificial Pancreas for Overnight Glucose Control in Type 1 Diabetes. J Clin Endocrinol Metab. 2016;101(1):214-23.

36. Haidar A, Legault L, Dallaire M, Alkhateeb A, Coriati A, Messier V, et al. Glucose-responsive insulin and glucagon delivery (dual-hormone artificial pancreas) in adults with type 1 diabetes: a randomized crossover controlled trial. Can Med Assoc J. 2013 Jan;185(4):297305.

37. El-Khatib FH, Russell SJ, Magyar KL, Sinha M, McKeon K, Nathan DM, et al. Autonomous and continuous adaptation of a bihormonal bionic pancreas in adults and adolescents with type 1 diabetes. J Clin Endocrinol Metab. 2014;99(5):1701-11.

38. Haidar A, Legault L, Messier V, Mitre TM, Leroux C, Rabasa-Lhoret R. Comparison of dualhormone artificial pancreas, single-hormone artificial pancreas, and conventional insulin pump therapy for glycaemic control in patients with type 1 diabetes: an open-label randomised controlled crossover trial. lancet Diabetes Endocrinol. 2015;3(1):17-26.

39. Taleb N, Emami A, Suppere C, Messier V, Legault L, Ladouceur M, et al. Efficacy of singlehormone and dual-hormone artificial pancreas during continuous and interval exercise in adult patients with type 1 diabetes: randomised controlled crossover trial. Diabetologia. 2016;59(12):2561-71.

40. Haidar A, Messier V, Legault L, Ladouceur M, Rabasa-Lhoret R. Outpatient 60-hour dayand-night glucose control with dual-hormone artificial pancreas, single-hormone artificial pancreas, or sensor-augmented pump therapy in adults with type 1 diabetes: An openlabel, randomised, crossover, controlled trial. Diabetes, Obes Metab. 2017;19(5):713-20. 
41. Haidar A, Smaoui MR, Legault L, Rabasa-Lhoret R. The role of glucagon in the artificial pancreas. Lancet Diabetes Endocrinol. 2016;4(6):476-9.

42. Bequette BW. Fault Detection and Safety in Closed-Loop Artificial Pancreas Systems. J Diabetes Sci Technol. 2014;8(6):1204-14.

43. Heinemann L, Krinelke L. Insulin Infusion Set: The Achilles Heel of Continuous Subcutaneous Insulin Infusion. J Diabetes Sci Technol. 2012;6(4):954-64.

44. de Vries L, Grushka Y, Lebenthal Y, Shalitin S, Phillip M. Factors associated with increased risk of insulin pump discontinuation in pediatric patients with type 1 diabetes. Pediatr Diabetes. 2011;12(5):506-12.

45. Blauw H, Keith-Hynes P, Koops R, DeVries JH. A Review of Safety and Design Requirements of the Artificial Pancreas. Ann Biomed Eng. 2016;1-15.

46. Joubert M, Reznik Y. Personal continuous glucose monitoring (CGM) in diabetes management: Review of the literature and implementation for practical use. Diabetes Res Clin Pract. 2012;96(3):294-305.

47. Schmidt S, Boiroux D, Duun-Henriksen AK, Frossing L, Skyggebjerg O, Jorgensen JB, et al. Model-Based Closed-Loop Glucose Control in Type 1 Diabetes: The DiaCon Experience. J Diabetes Sci Technol. 2013;7(5):1255-64.

48. Ly TT, Roy A, Grosman B, Shin J, Campbell A, Monirabbasi S, et al. Day and Night ClosedLoop Control Using the Integrated Medtronic Hybrid Closed-Loop System in Type 1 Diabetes at Diabetes Camp. Diabetes Care. 2015;38(7):1205-11.

49. Plougmann S, Hejlesen O, Turner B, Kerr D, Cavan D. The effect of alcohol on blood glucose in Type 1 diabetes-metabolic modelling and integration in a decision support system. Int J Med Inform. 2003;70(2-3):337-44.

50. Turner BC, Jenkins E, Kerr D, Sherwin RS, Cavan DA. The Effect of Evening Alcohol Consumption on Next-Morning Glucose Control in Type 1 Diabetes. Diabetes Care. 
$2001 ; 24(11): 1888-93$.

51. Richardson T, Weiss M, Thomas P, Kerr D. Day After the Night Before: Influence of evening alcohol on risk of hypoglycemia in patients with type 1 diabetes. Diabetes Care. 2005;28(7):1801-2.

52. Hovorka R, Kumareswaran K, Harris J, Allen JM, Elleri D, Xing D, et al. Overnight closed loop insulin delivery (artificial pancreas) in adults with type 1 diabetes: crossover randomised controlled studies. BMJ. 2011;342:1-10.

53. Kovatchev BP, Renard E, Cobelli C, Zisser HC, Keith-Hynes P, Anderson SM, et al. Safety of outpatient closed-loop control: First randomized crossover trials of a wearable artificial pancreas. Diabetes Care. 2014;37(7):1789-96.

54. Gingras V, Haidar A, Messier V, Legault L, Ladouceur M, Rabasa-Lhoret R. A Simplified Semiquantitative Meal Bolus Strategy Combined with Single- and Dual-Hormone ClosedLoop Delivery in Patients with Type 1 Diabetes: A Pilot Study. Diabetes Technol Ther. 2016;18(8):464-71 .

55. van Bon AC, Luijf YM, Koebrugge R, Koops R, Hoekstra JBL, DeVries JH. Feasibility of a Portable Bihormonal Closed-Loop System to Control Glucose Excursions at Home Under Free-Living Conditions for 48 Hours. Diabetes Technol Ther. 2014 Mar;16(3):131-6.

56. Walter KN, Wagner JA, Cengiz E, Tamborlane W V, Petry NM. The need for research addressing alcohol use disorder and diabetes. Addiction. 2016;111(5):763-5.

57. Murphy HR, Rayman G, Duffield K, Lewis KS, Kelly S, Johal B, et al. Changes in the Glycemic Profiles of Women With Type 1 and Type 2 Diabetes During Pregnancy. Diabetes Care. 2007;30(11):2785-91.

58. Murphy HR, Elleri D, Allen JM, Harris J, Simmons D, Rayman G, et al. Closed-Loop Insulin Delivery During Pregnancy Complicated by Type 1 Diabetes. Diabetes Care. $2011 ; 34(2): 406-11$. 
59. Murphy HR, Kumareswaran K, Elleri D, Allen JM, Caldwell K, Biagioni M, et al. Safety and Efficacy of 24-h Closed-Loop Insulin Delivery in Well-Controlled Pregnant Women With Type 1 Diabetes: A randomized crossover case series. Diabetes Care. 2011;34(12):25279.

60. Stewart ZA, Wilinska ME, Hartnell S, Temple RC, Rayman G, Stanley KP, et al. ClosedLoop Insulin Delivery during Pregnancy in Women with Type 1 Diabetes. N Engl J Med. 2016;375(7):644-54.

61. Jeffery AN, Metcalf BS, Hosking J, Streeter AJ, Voss LD, Wilkin TJ. Age Before Stage: Insulin Resistance Rises Before the Onset of Puberty. Diabetes Care. 2012;35(3):536-41.

62. Nimri R, Danne T, Kordonouri O, Atlas E, Bratina N, Biester T, et al. The "Glucositter" overnight automated closed loop system for type 1 diabetes: A randomized crossover trial. Pediatr Diabetes. 2013;14(3):159-67.

63. Nimri R, Muller I, Atlas E, Miller S, Fogel A, Bratina N, et al. MD-logic overnight control for 6 weeks of home use in patients with type 1 diabetes: Randomized crossover trial. Diabetes Care. 2014;37(11):3025-32.

64. Beck RW, Tamborlane W V, Bergenstal RM, Miller KM, DuBose SN, Hall CA. The T1D Exchange Clinic Registry. J Clin Endocrinol Metab. 2012;97(12):4383-9.

65. Elleri D, Maltoni G, Allen JM, Nodale M, Kumareswaran K, Leelarathna L, et al. Safety of closed-loop therapy during reduction or omission of meal boluses in adolescents with type 1 diabetes: a randomized clinical trial. Diabetes, Obes Metab. 2014;16(11):1174-8.

66. Maahs DM, Buckingham BA, Castle JR, Cinar A, Damiano ER, Dassau E, et al. Outcome Measures for Artificial Pancreas Clinical Trials: A Consensus Report. Diabetes Care. 2016;39(7):1175-9.

67. Thabit $\mathrm{H}$, Tauschmann M, Allen JM, Leelarathna L, Hartnell S, Wilinska ME, et al. Home Use of an Artificial Beta Cell in Type 1 Diabetes. N Engl J Med. 2015;373(22):2129-40. 
68. Kropff J, Favero S Del, Place J, Toffanin C, Visentin R, Monaro M, et al. 2 month evening and night closed-loop glucose control in patients with type 1 diabetes under free-living conditions: a randomised crossover trial. Lancet Diabetes Endocrinol. 2015;3(12):939-47.

69. Pinsker JE, Lee JB, Dassau E, Seborg DE, Bradley PK, Gondhalekar R, et al. Randomized Crossover Comparison of Personalized MPC and PID Control Algorithms for the Artificial Pancreas. Diabetes Care. 2016;39(7):1135-42.

70. Doyle FJ, Huyett LM, Lee JB, Zisser HC, Dassau E. Closed-Loop Artificial Pancreas Systems: Engineering the Algorithms. Diabetes Care. 2014;37(5):1191-7. 